\title{
Structure of Exotic Nuclei: A Theoretical Review
}

\author{
Shan-Gui Zhou* \\ CAS Key Laboratory of Frontiers in Theoretical Physics, Institute of Theoretical Physics, \\ Chinese Academy of Sciences, Beijing 100190, China \\ School of Physical Sciences, University of Chinese Academy of Sciences, Beijing 100049, China \\ Center of Theoretical Nuclear Physics, National Laboratory of Heavy Ion Accelerator, Lanzhou \\ 730000, China \\ Synergetic Innovation Center for Quantum Effects and Application, Hunan Normal University, \\ Changsha, 410081, China
}

E-mail: sqzhouditp.ac.cn

The study of exotic nuclei-nuclei with the ratio of neutron number $N$ to proton number $Z$ deviating much from that of those found in nature-is at the forefront of nuclear physics research because it can not only reveal novel nuclear properties and thus enrich our knowledge of atomic nuclei, but also help us to understand the origin of chemical elements in the nucleosynthesis. With the development of radioactive ion beam facilities around the world, more and more unstable nuclei become experimentally accessible. Many exotic nuclear phenomena have been observed or predicted in nuclei far from the $\beta$-stability line, such as neutron or proton halos, the shell evolution and changes of nuclear magic numbers, the island of inversion, soft-dipole excitations, clustering effects, new radioactivities, giant neutron halos, the shape decoupling between core and valence nucleons in deformed halo nuclei, etc. In this contribution, I will present a review of theoretical study of exotic nuclear structure. I will first introduce characteristic features and new physics connected with exotic nuclear phenomena: the weakly-bound feature, the large-spatial extension in halo nuclei, deformation effects in halo nuclei, the shell evolution, new radioactivities and clustering effects. Then I will highlight some recent progresses corresponding to these features.

The 26th International Nuclear Physics Conference

11-16 September, 2016

Adelaide, Australia

* Supported by the NSF of China (11275248, 11525524, 11621131001 and 11647601), the 973 Program of China (2013CB834400), the Key Research Program of Frontier Sciences of CAS, the HPC Cluster of SKLTP/ITP-CAS and the Supercomputing Center, CNIC of CAS. 


\section{Introduction}

In nature, there are less than three hundred stable or long-lived nuclides which are along the valley of stability in the nuclear chart. When those unstable nuclei, the number is now close to three thousand [四, are explored, many exotic nuclear phenomena have been observed. The most famous exotic nucleus is ${ }^{11} \mathrm{Li}$ in which the halo structure was identified [0]. Theoretically, many more nuclei are predicted to be bound. In Fig. W is shown the prediction from the Weiszacker-Skyrme (WS4) mass model [ [3] which is one of the best nuclear mass models on the market. Many other

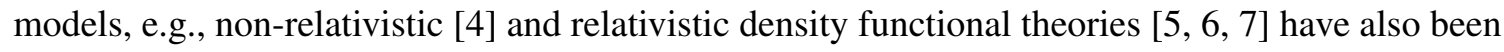
used to explore the border of nuclear chart. Nowadays, the study of the properties of these exotic nuclei is at the forefront of nuclear physics research because it can not only reveal new physics but also lead to new insights on the nucleosynthesis.

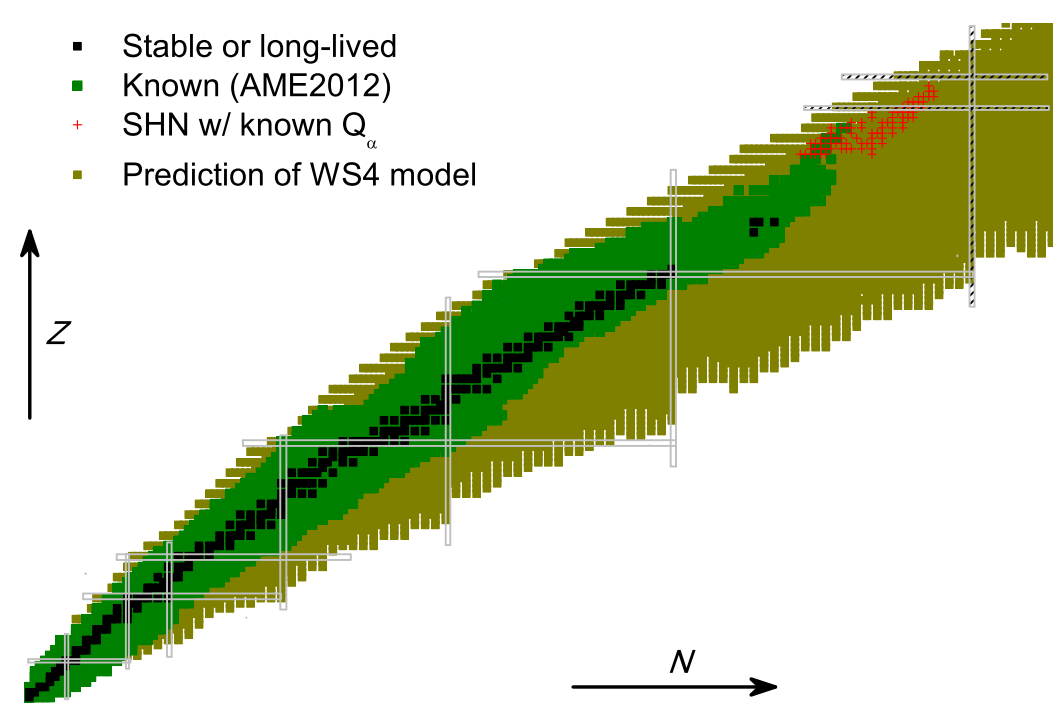

Figure 1: (Color online) Nuclear chart consisting of stable or long-lived nuclides, known and unstable

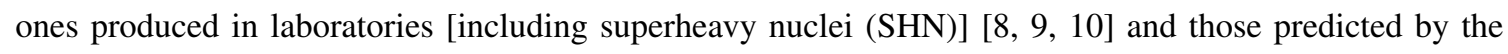
Weiszacker-Skyrme (WS4) mass model [3]. Courtesy of Ning Wang.

In this contribution, I will first discuss the physics connected with exotic nuclear phenomena in Section \. According to my personal point of view, six features will be illustrated concerning exotic nuclear structure. Then I'll highlight some recent progresses corresponding to each of these features in Section [3. Finally I will discuss perspectives in Section 6 .

\section{Physics in exotic nuclear structure}

The first important characteristic of exotic nuclei is certainly the weakly-bound feature. In unstable nuclei, particularly in those close to drip lines, e.g., to the neutron drip line, the neutron

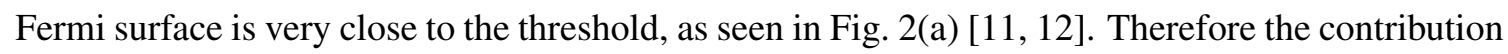
from continua becomes more and more important. In Fig. Z(b), the neutron separation energy $S_{n}$, equivalent to the neutron Fermi energy, is shown schematically as a function of neutron number 
[[13], [4]]. Larger neutron excess results in smaller $S_{n}$, i.e., the valence neutron(s) is (are) more easily knocked out and the nucleus is more easily coupled to the scattering environment, thus making exotic nuclei open quantum systems which are very much involved in the studies of nuclear reactions and nucleosynthesis.

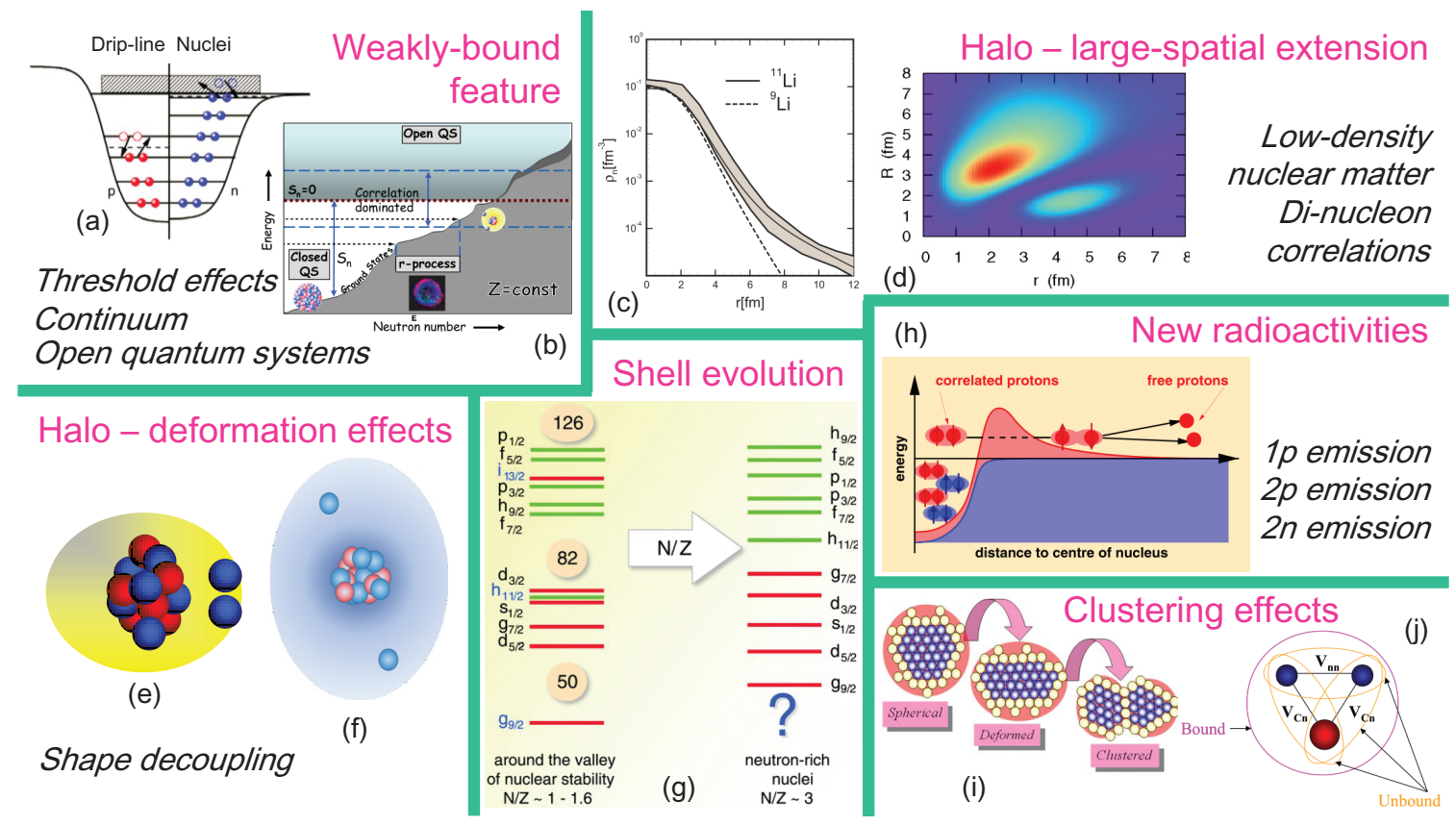

Figure 2: (Color online) Physics in exotic nuclear structure. (a), (b), (c), (d), (g), (h), (i) and (j) are taken

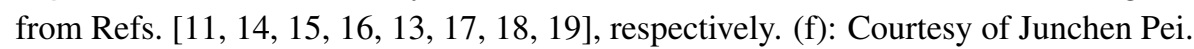

Halo nuclei are characterized by a large spatial extension, see Fig. $\left[\right.$ (c) for an example for ${ }^{11} \mathrm{Li}$ [ㄷ]]. In neutron halo nuclei, there appears pure neutron matter with a very low density, surrounding a dense core [ㄴ], [2], [5, [20]. Similar to what happens in low density infinite nuclear matter or neutron matter [2], [22, [23], pair condensate or strong di-neutron correlations may occur in finite nuclei with halo structure [Fig. I(d)] [ए6, [24]. In addition, the oscillation between the core and the low density neutron matter leads to some soft dipole modes, also known as pygmy dipole resonances, which have been discussed a lot in INPC2016. Experimentally these features have been explored in, e.g., Refs. [25, [26].

Most known nuclei are deformed [D7]. What kind of new features can deformation effects bring to exotic nuclei, in particular, to halo nuclei? Note that in recent years, more candidates of deformed halo nuclei have been identified; examples are ${ }^{31} \mathrm{Ne}\left[[28]\right.$ and ${ }^{37} \mathrm{Mg}$ [R2]. For deformation effects in halo nuclei, I will focus on theoretical predictions on the shape decoupling [Fig. D(e) \& (f)] [B], B], B2], [33].

Shell structure is very important in the study of atomic nuclei which is characterized by large spin-orbit couplings. The spin-orbit couplings are closely connected with the nuclear surface diffuseness. It is therefore very natural that the spin-orbit splitting would change when going from the $\beta$-stability line to the drip lines because the nuclear surface could be more diffuse [Fig. $\square(\mathrm{g})$ ] [[3]]. This results in the shell evolution in exotic nuclei and changes of nuclear magicity which 
can be hinted from, e.g., separation energies [34]. Certainly the shell evolution is also the result of many other important factors, like the tensor force [35], 36, 373, 38, [39]. It should be emphasized that shape evolution and shape coexistence are also related physical topics of exotic nuclei, which were discussed in a dedicated session in INPC2016.

Beyond the drip lines, nuclei are unbound with respect to nucleon(s) emission. This feature implies some new radioactivities of which mostly discussed are one- or two-proton radioactivities, thanks to the Coulomb interaction which leads to a Coulomb barrier hindering the escape of pro-

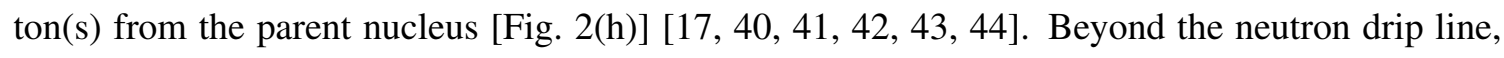
there may be the two-neutron radioactivity; one example of recent interests is ${ }^{26} \mathrm{O}$ [45], 46, 47]].

It is well known that clustering effects are important in atomic nuclei and cluster structure appears in some stable ones if they are excited to be close to some thresholds [48]. In exotic nuclei, clustering effects can also emerge in low-lying excited states and even in ground states. For exotic nuclei with much more neutrons, a cluster configuration is energetically more favored because it permits a more even distribution of valence neutrons as shown in Fig. ए(i) [ए8]. In one recent

experimental study of ${ }^{12} \mathrm{Be}$, a $0^{+}$resonant state with a large cluster decay branching ratio was observed [49]. This observation supports strong clustering effects in ${ }^{12} \mathrm{Be}$. In addition, in some

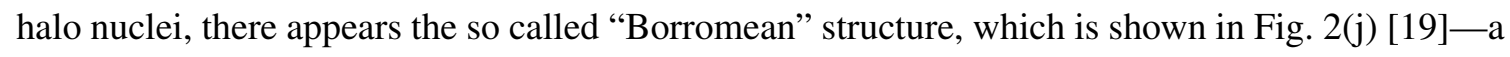
bound three-body system with any two-body subsystems unbound.

\section{Highlights of recent progresses}

Next I will highlight some recent progresses on theoretical study of exotic nuclear structure. There are indeed many interesting and important works, but I can only choose some of them due to the limitation of pages. More extensive discussions can be found in Ref. [50].

\subsection{The weakly bound feature of exotic nuclei}

Many models have been developed to take into account the contribution of continua and resonances, see, e.g., Refs. [[1], [24, [1], [2], [5] for recent reviews. There are many ways to locate single particle resonances. Besides the conventional scattering phase shift method [54], several bound-state-like approaches [ [55, 56], such as the analytical continuation in coupling constant [57, 58, 59, 60, 61, 62] , the real stabilization method [63, 64, 65] and the complex scaling method (CSM) [66, 67, 68, 69], are often used to study single particle resonances in atomic nuclei. Sev-

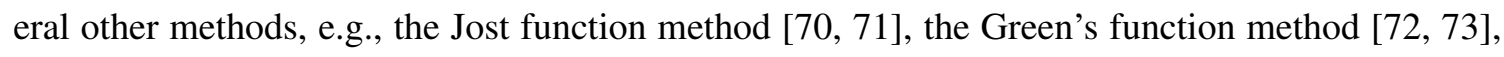
the Green's function + CSM [69, [4]], and solving Schrödinger or Dirac equations in the complex momentum representation [ [75, [6], have also been implemented in nuclear models.

For describing the contribution from the continua in the mean field level, the conventional BCS method suffers from some problems. One of them is the non-localization of nucleon density distributions [[Z], [8]]. One way to solve partly this problem is to use the resonance BCS (rBCS) approach [ [79, [0], [1]]: After single particle resonances are located, their contribution can be taken into account through the BCS approximation. It was also justified that if one, instead of using the conventional BCS approximation, makes Bogoliubov transformation and solves the Hartree-FockBogoliubov (HFB) equations in $r$ space, the contribution from continua can be included properly and the nucleus in question is localized [ㄱ, [8]]. Since then, the HFB models have been developed 
for spherical nuclei with continuum either discretized [18], 82, [8], [84] or treated with scattering boundary conditions [ [85, [86, [87]. The HFB model was also extended to the study of deformed nuclei [32], 33], 88]]. In parallel, the relativistic Hartree-Bogoliubov (RHB) or relativistic HFB

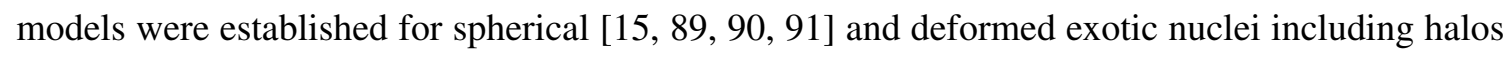
[B], B], [2], [23].

\subsection{Deformation effects in nuclear halos}

Based on the deformed RHB model in a Woods-Saxon basis [94], shape decoupling effects have been predicted in ${ }^{42,44} \mathrm{Mg}$ [30, $\left.\mathrm{B}\right]$ ]: The core of these nuclei is prolate, but the halo has an oblate shape. The generic conditions for the occurrence of halo in deformed nuclei and shape decoupling effects were given in Ref. [B]]. Later, with a non-relativistic HFB model, Pei et al. predicted that ${ }^{38} \mathrm{Ne}$ has a nearly spherical core, but a prolate halo [B2], [33]]. Similar effects has been investigated in Ref. [05] in which a square well potential was used and the spin-orbit coupling was neglected. These predictions are made for the ground state. It would be interesting to study dynamics and excitations of these deformed halo nuclei [06].

\subsection{Di-neutron correlations}

Concerning di-neutron correlations, progresses in recent years include the study of Cooper pairs [97], di-neutron correlations [98], di-proton correlations [99], neutron-proton correlations [ए00], and so on. For example, the asymptotic form of a neutron Cooper pair penetrating to the exterior of the nuclear surface was investigated with the Bogoliubov theory in Ref. [Q7]]. It was found that Cooper pairs are spatially correlated in the asymptotic large distance limit, and the penetration length of the pair condensate is universally governed by the two-neutron separation energy.

There are also lots of theoretical investigations on the soft dipole modes [ए0], ㅁ02, 0.3, ए04,

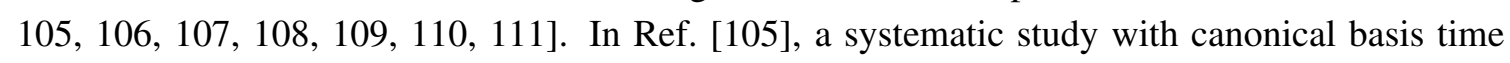
dependent HFB theory reveals a number of characteristic features of the low-energy $E 1$ modes, e.g., a universal behavior in the low-energy $E 1$ modes for heavy neutron-rich isotopes, which suggests the emergence of decoupled $E 1$ peaks beyond $N=82$.

\subsection{The shell evolution}

It is interesting and instructive to choose the Ca isotope chain as an example to discuss the evolution of shell structure and changes of magicity because there might be five magic numbers in the Ca isotopes. In Ref. [1]2], from the measured mass of ${ }^{53,54} \mathrm{Ca}$, a prominent shell closure at $N=32$ was established. This shell closure was later confirmed together with a new one at $N=34$, indicated by the fact that the energy of the first $2^{+}$state for ${ }^{52,54} \mathrm{Ca}$ rises dramatically [ए13]. Recently the magicity of $N=32$ was shown to persist in Sc isotopes [14]. The appearance of the shell closures at $N=32$ and 34 have been attributed to the evolution of neutron $f_{5 / 2}$ orbital, which rises due to a weakened proton-neutron interaction when $Z$ decreases to 20 [ए3]].

Theoretically there have been many investigations on the magicity of $N=32$ and/or 34 [ए5], ए6, ए7, ए8]. For example, it has been shown in Ref. [15] that the like-particle tensor contribution is responsible for these new shell closures and in Ref. [ए18], the importance of exchange 
terms in relativistic framework on these shell closures was emphasized. However, a recent precise measurement of charge radii in Ca isotopes [एव] casts some doubts on the magicity at $N=32$. If ${ }^{52} \mathrm{Ca}$ is doubly magic, its charge radius should be smaller than that of its neighbors. But this is not the case [एव]]. Therefore nuclear magicity in exotic nuclei may be "local" in the sense that it manifests itself in some nuclear properties but not in others, contrast to those traditional magic numbers which are "global" or robust and manifest themselves in "all" nuclear properties, e.g., separation energies, charge radii, $Q$ values of $\alpha$ decays, etc.

\subsection{New radioactivities}

For new radioactivity, without going into details, I'd like to mention that there have been some systematic studies with the HFB model [ㄴ20] and predictions were also made with the relativistic continuum Hartree-Bogoliubov model [ए2]].

\subsection{Clustering effects}

There have been many interesting results concerning the theoretical study of clustering effects in atomic nuclei. For example, a non-localized or container picture was proposed for cluster structure [122], giant dipole resonances were argued to be a fingerprint of cluster structure [[123], one-dimensional $\alpha$ condensation of $\alpha$-linear-chain states in ${ }^{12} \mathrm{C}$ and ${ }^{16} \mathrm{O}$ were studied [ए24] and rod-shaped nuclei were explored at extremely high spin and isospin [ए25].

One more thing about clustering effects is that from radius-constrained mean field calculations, regardless of non-relativistic [126] or relativistic models [1227, [28], one can also obtain the cluster structure. However, in such kind of studies, one has to take a serious care of the truncation of the basis and to ensure a convergence [120].

\section{Concluding remarks and perspectives}

To summarize, after introducing the following characteristic features and new physics connected with exotic nuclear phenomena: the weakly-bound feature, the large-spatial extension in halo nuclei, deformation effects in halo nuclei, the shell evolution, new radioactivities and clustering effects, I have highlighted some recent progresses corresponding to these features.

It should be emphasized that to describe the structure of exotic nuclei, one often needs to modify conventional nuclear models or develop new theoretical approaches. Nowadays, there are many attempts to unify nuclear models. For example, with the fast development of supercomputers, ab initio theories can deal with heavier and heavier nuclei, as discussed in Ekström and Bacca's talks. Besides that, there are also projects to develop density functional theories from first principles [130, [13]] (also mentioned in Liang's plenary talk) or models based on subnucleon degrees of freedom [ए132].

In his talk, Nazarewicz has put atomic nuclei on a table with three pillars. This table would not be stable if it had only two pillars, only theory and simulations. We need experiments and experimental facilities. With the development of radioactive ion beam facilities around the world, including the High Intensity heavy ion Accelerator Facility (HIAF) in Huizhou, China [ए133, [134] 
and Beijing Isotope-Separation-On-Line Neutron-Rich Beam Facility (BISOL) [ए35], more unstable nuclei would become experimentally accessible, which will for sure challenge as well as provide opportunities for theoretical study of exotic nuclear structure.

\section{Acknowledgements}

Collaborations and/or helpful discussions with A. Afanasjev, K. Blaum, Y. Chen, G. Colo, L.S. Geng, N.V. Giai, L.L. Li, H.Z. Liang, W.H. Long, B.N. Lu, H.F. Lü, J. Meng, J. Pei, P. Ring, H. Sagawa, J.R. Stone, X.X. Sun, I. Tanihata, J. Terasaki, A.W. Thomas, H. Toki, D. Vretenar, N. Wang, F. R. Xu, S. Yamaji, J.Y. Zeng, Y.H. Zhang, S.Q. Zhang, E.G. Zhao, J. Zhao and P.W. Zhao are gratefully acknowledged.

\section{References}

[1] M. Thoennessen, Rep. Prog. Phys. 76, 056301 (2013)

[2] I. Tanihata, H. Hamagaki, O. Hashimoto, Y. Shida, N. Yoshikawa, K. Sugimoto, O. Yamakawa, T. Kobayashi, N. Takahashi, Phys. Rev. Lett. 55, 2676 (1985)

[3] N. Wang, M. Liu, X. Wu, J. Meng, Phys. Lett. B 734, 215 (2014)

[4] J. Erler, N. Birge, M. Kortelainen, W. Nazarewicz, E. Olsen, A.M. Perhac, M. Stoitsov, Nature 486, $509(2012)$

[5] A.V. Afanasjev, S.E. Agbemava, D. Ray, P. Ring, Phys. Lett. B 726, 680 (2013)

[6] X. Qu, Y. Chen, S. Zhang, P. Zhao, I. Shin, Y. Lim, Y. Kim, J. Meng, Sci. China-Phys. Mech. Astron. 56, 2031 (2013)

[7] K.Q. Lu, Z.X. Li, Z.P. Li, J.M. Yao, J. Meng, Phys. Rev. C 91, 027304 (2015)

[8] G. Audi, F.G. Kondev, M. Wang, B. Pfeiffer, X. Sun, J. Blachot, M. MacCormick, Chin. Phys. C 36, 1157 (2012)

[9] G. Audi, M. Wang, A.H. Wapstra, F.G. Kondev, M. MacCormick, X. Xu, B. Pfeiffer, Chin. Phys. C 36, 1287 (2012)

[10] M. Wang, G. Audi, A.H. Wapstra, F.G. Kondev, M. MacCormick, X. Xu, B. Pfeiffer, Chin. Phys. C 36, 1603 (2012)

[11] J. Meng, H. Toki, S.G. Zhou, S.Q. Zhang, W.H. Long, L.S. Geng, Prog. Part. Nucl. Phys. 57, 470 (2006)

[12] J. Meng, S.G. Zhou, J. Phys. G: Nucl. Part. Phys. 42, 093101 (2015)

[13] J. Dobaczewski, N. Michel, W. Nazarewicz, M. Ploszajczak, J. Rotureau, Prog. Part. Nucl. Phys. 59, 432 (2007)

[14] N. Michel, W. Nazarewicz, M. Ploszajczak, T. Vertse, J. Phys. G: Nucl. Phys. 36, 013101 (2009)

[15] J. Meng, P. Ring, Phys. Rev. Lett. 77, 3963 (1996)

[16] K. Hagino, H. Sagawa, J. Carbonell, P. Schuck, Phys. Rev. Lett. 99, 022506 (2007)

[17] B. Blank, M. Ploszajczak, Rep. Prog. Phys. 71, 046301 (2008)

[18] M. Freer, Rep. Prog. Phys. 70, 2149 (2007) 
[19] B. Johnson, Phys. Rep. 389, 1 (2004)

[20] D. Vretenar, A.V. Afanasjev, G.A. Lalazissis, P. Ring, Phys. Rep. 409, 101 (2005)

[21] B.Y. Sun, H. Toki, J. Meng, Phys. Lett. B 683, 134 (2010)

[22] M. Matsuo, Phys. Rev. C 73, 044309 (2006)

[23] T.T. Sun, B.Y. Sun, J. Meng, Phys. Rev. C 86, 014305 (2012)

[24] H. Sagawa, K. Hagino, Eur. Phys. J. A 51, 102 (2015)

[25] T. Nakamura, A.M. Vinodkumar, T. Sugimoto et al., Phys. Rev. Lett. 96, 252502 (2006)

[26] R. Kanungo, A. Sanetullaev, J. Tanaka et al., Phys. Rev. Lett. 114, 192502 (2015)

[27] S.G. Zhou, Phys. Scr. 91, 063008 (2016)

[28] T. Nakamura, N. Kobayashi, Y. Kondo et al., Phys. Rev. Lett. 112, 142501 (2014)

[29] N. Kobayashi, T. Nakamura, Y. Kondo et al., Phys. Rev. Lett. 112, 242501 (2014)

[30] S.G. Zhou, J. Meng, P. Ring, E.G. Zhao, Phys. Rev. C 82, 011301(R) (2010)

[31] L. Li, J. Meng, P. Ring, E.G. Zhao, S.G. Zhou, Phys. Rev. C 85, 024312 (2012)

[32] J.C. Pei, Y.N. Zhang, F.R. Xu, Phys. Rev. C 87, 051302(R) (2013)

[33] J.C. Pei, G.I. Fann, R.J. Harrison, W. Nazarewicz, Y. Shi, S. Thornton, Phys. Rev. C 90, 024317 (2014)

[34] A. Ozawa, T. Kobayashi, T. Suzuki, K. Yoshida, I. Tanihata, Phys. Rev. Lett. 84, 5493 (2000)

[35] S. Peru, M. Girod, J. Berger, Eur. Phys. J. A 9, 35 (2000)

[36] T. Otsuka, T. Suzuki, R. Fujimoto, H. Grawe, Y. Akaishi, Phys. Rev. Lett. 95, 232502 (2005)

[37] G. Colo, H. Sagawa, S. Fracasso, P. Bortignon, Phys. Lett. B 646, 227 (2007)

[38] O. Sorlin, M.G. Porquet, Prog. Part. Nucl. Phys. 61, 602 (2008)

[39] H. Sagawa, G. Colo, Prog. Part. Nucl. Phys. 76, 76 (2014)

[40] P.J. Woods, C.N. Davids, Annu. Rev. Nucl. Part. Sci. 47, 541 (1997)

[41] M. Thoennessen, Rep. Prog. Phys. 67, 1187 (2004)

[42] C. Lin, X. Xu, H. Jia et al., Sci. China-Phys. Mech. Astron. 54 (Suppl. 1), 73 (2011)

[43] M. Pfutzner, M. Karny, L.V. Grigorenko, K. Riisager, Rev. Mod. Phys. 84, 567 (2012)

[44] Y.G. Ma, D.Q. Fang, X.Y. Sun et al., Phys. Lett. B 743, 306 (2015)

[45] E. Lunderberg, P.A. DeYoung, Z. Kohley et al., Phys. Rev. Lett. 108, 142503 (2012)

[46] Z. Kohley, T. Baumann, D. Bazin et al., Phys. Rev. Lett. 110, 152501 (2013)

[47] Y. Kondo, T. Nakamura, R. Tanaka et al., Phys. Rev. Lett. 116, 102503 (2016)

[48] W. von Oertzen, M. Freer, Y. Kanada-En'yo, Phys. Rep. 432, 43 (2006)

[49] Z.H. Yang, Y.L. Ye, Z.H. Li et al., Phys. Rev. Lett. 112, 162501 (2014)

[50] S.G. Zhou, Theoretical Study of Exotic Nuclear Structure, in Nuclear Structure in China 2016 Proceedings of the 16th National Conference on Nuclear Structure in China, to be published 
[51] T. Frederico, A. Delfino, L. Tomio, M.T. Yamashita, Prog. Part. Nucl. Phys. 67, 939 (2012)

[52] C. Ji, Int. J. Mod. Phys. E 25, 1641003 (2016)

[53] J. Meng, P. Ring, P. Zhao, S.G. Zhou, Relativistic mean field description of exotic nuclei, Chap. 3 in Vol. 10 of International Review of Nuclear Physics (World Scientific Publishing Co. Pte. Ltd., 2016, edited by J. Meng), pp. 83-141

[54] I. Hamamoto, Phys. Rev. C 93, 054328 (2016)

[55] V.D. Efros, W. Leidemann, G. Orlandini, N. Barnea, J. Phys. G: Nucl. Part. Phys. 34, R459 (2007)

[56] J. Carbonell, A. Deltuva, A.C. Fonseca, R. Lazauskas, Prog. Part. Nucl. Phys. 74, 55 (2014)

[57] N. Tanaka, Y. Suzuki, K. Varga, Phys. Rev. C 56, 562 (1997)

[58] S.C. Yang, J. Meng, S.G. Zhou, Chin. Phys. Lett. 18, 196 (2001)

[59] S.S. Zhang, J. Meng, S.G. Zhou, G.C. Hillhouse, Phys. Rev. C 70, 034308 (2004)

[60] J.Y. Guo, X.Z. Fang, Phys. Rev. C 74, 024320 (2006)

[61] S.S. Zhang, M.S. Smith, G. Arbanas, R.L. Kozub, Phys. Rev. C 86, 032802 (2012)

[62] X.D. Xu, S.S. Zhang, A.J. Signoracci, M.S. Smith, Z.P. Li, Phys. Rev. C 92, 024324 (2015)

[63] L. Zhang, S.G. Zhou, J. Meng, E.G. Zhao, Phys. Rev. C 77, 014312 (2008)

[64] S.G. Zhou, J. Meng, E.G. Zhao, J. Phys. B: At. Mol. Phys. 42, 245001 (2009)

[65] J.C. Pei, A.T. Kruppa, W. Nazarewicz, Phys. Rev. C 84, 024311 (2011)

[66] T. Myo, Y. Kikuchi, H. Masui, K. Katō, Prog. Part. Nucl. Phys. 79, 1 (2014)

[67] G. Papadimitriou, J.P. Vary, Phys. Rev. C 91, 021001(R) (2015)

[68] M. Shi, Q. Liu, Z.M. Niu, J.Y. Guo, Phys. Rev. C 90, 034319 (2014)

[69] M. Shi, J.Y. Guo, Q. Liu, Z.M. Niu, T.H. Heng, Phys. Rev. C 92, 054313 (2015)

[70] B.N. Lu, E.G. Zhao, S.G. Zhou, Phys. Rev. Lett. 109, 072501 (2012)

[71] B.N. Lu, E.G. Zhao, S.G. Zhou, Phys. Rev. C 88, 024323 (2013)

[72] M. Matsuo, Nucl. Phys. A 696, 371 (2001)

[73] T.T. Sun, S.Q. Zhang, Y. Zhang, J.N. Hu, J. Meng, Phys. Rev. C 90, 054321 (2014)

[74] X.X. Shi, M. Shi, Z.M. Niu, T.H. Heng, J.Y. Guo, Phys. Rev. C 94, 024302 (2016)

[75] N. Li, M. Shi, J.Y. Guo, Z.M. Niu, H. Liang, Phys. Rev. Lett. 117, 062502 (2016)

[76] Z. Fang, M. Shi, J.Y. Guo, Z.M. Niu, H. Liang, S.S. Zhang, Phys. Rev. C 95, 024311 (2017)

[77] A. Bulgac, IPNE FT-194-1980, Bucharest (arXiv: nucl-th/9907088)

[78] J. Dobaczewski, H. Flocard, J. Treiner, Nucl. Phys. A 422, 103 (1984)

[79] N. Sandulescu, N. Van Giai, R.J. Liotta, Phys. Rev. C 61, 061301(R) (2000)

[80] L. Geng, H. Toki, S. Sugimoto, J. Meng, Prog. Theo. Phys. 110, 921 (2003)

[81] S.S. Zhang, E.G. Zhao, S.G. Zhou, Euro. Phys. J. A 49, 77 (2013)

[82] J. Dobaczewski, W. Nazarewicz, T.R. Werner, J.F. Berger, C.R. Chinn, J. Dechargé, Phys. Rev. C 53, 2809 (1996) 
[83] Y. Yu, A. Bulgac, Phys. Rev. Lett. 90, 222501 (2003)

[84] N. Schunck, J.L. Egido, Phys. Rev. C 78, 064305 (2008)

[85] Y. Zhang, M. Matsuo, J. Meng, Phys. Rev. C 83, 054301 (2011)

[86] Y. Zhang, M. Matsuo, J. Meng, Phys. Rev. C 86, 054318 (2012)

[87] Y. Zhang, Y. Chen, J. Meng, P. Ring, Phys. Rev. C 95, 014316 (2017)

[88] H. Nakada, Nucl. Phys. A 808, 47 (2008)

[89] J. Meng, Nucl. Phys. A 635, 3 (1998)

[90] W. Pöschl, D. Vretenar, G.A. Lalazissis, P. Ring, Phys. Rev. Lett. 79, 3841 (1997)

[91] W.H. Long, P. Ring, N.V. Giai, J. Meng, Phys. Rev. C 81, 024308 (2010)

[92] L. Li, J. Meng, P. Ring, E.G. Zhao, S.G. Zhou, Chin. Phys. Lett. 29, 042101 (2012)

[93] Y. Chen, L. Li, H. Liang, J. Meng, Phys. Rev. C 85, 067301 (2012)

[94] S.G. Zhou, J. Meng, P. Ring, Phys. Rev. C 68, 034323 (2003)

[95] T. Misu, W. Nazarewicz, S. Åberg, Nucl. Phys. A 614, 44 (1997)

[96] K. Fossez, W. Nazarewicz, Y. Jaganathen, N. Michel, M. Płoszajczak, Phys. Rev. C 93, 011305(R) (2016)

[97] Y. Zhang, M. Matsuo, J. Meng, Phys. Rev. C 90, 034313(R) (2014)

[98] F. Kobayashi, Y. Kanada-En'yo, Phys. Rev. C 93, 024310 (2016)

[99] T. Oishi, K. Hagino, H. Sagawa, Phys. Rev. C 90, 034303 (2014)

[100] H. Masui, M. Kimura, Prog. Theor. Exp. Phys. 2016, 053 D01 (2016)

[101] N. Paar, D. Vretenar, G. Colo, Rep. Prog. Phys. 70, 691 (2007)

[102] X. Roca-Maza, G. Pozzi, M. Brenna, K. Mizuyama, G. Colò, Phys. Rev. C 85, 024601 (2012)

[103] D. Vretenar, Y.F. Niu, N. Paar, J. Meng, Phys. Rev. C 85, 044317 (2012)

[104] D. Savran, T. Aumann, A. Zilges, Prog. Part. Nucl. Phys. 70, 210 (2013)

[105] S. Ebata, T. Nakatsukasa, T. Inakura, Phys. Rev. C 90, 024303 (2014)

[106] T. Inakura, W. Horiuchi, Y. Suzuki, T. Nakatsukasa, Phys. Rev. C 89, 064316 (2014)

[107] P. Papakonstantinou, H. Hergert, R. Roth, Phys. Rev. C 92, 034311 (2015)

[108] H.L. Ma, B.G. Dong, Y.L. Yan, H.Q. Zhang, D.Q. Yuan, S.Y. Zhu, X.Z. Zhang, Phys. Rev. C 93, 014317 (2016)

[109] G. De Gregorio, F. Knapp, N. Lo Iudice, P. Vesely, Phys. Rev. C 93, 044314 (2016)

[110] H. Zheng, S. Burrello, M. Colonna, V. Baran, Phys. Rev. C 94, 014313 (2016)

[111] T. Nakatsukasa, K. Matsuyanagi, M. Matsuo, K. Yabana, Rev. Mod. Phys. 88, 045004 (2016)

[112] F. Wienholtz, D. Beck, K. Blaum et al., Nature 498, 346 (2013)

[113] D. Steppenbeck, S. Takeuchi, N. Aoi et al., Nature 502, 207 (2013)

[114] X. Xu, M. Wang, Y.H. Zhang et al., Chin. Phys. C 39, 104001 (2015) 
[115] M. Grasso, Phys. Rev. C 89, 034316 (2014)

[116] E. Yüksel, N. Van Giai, E. Khan, K. Bozkurt, Phys. Rev. C 89 (2014)

[117] X.B. Wang, G.X. Dong, J. Phys. G: Nucl. Part. Phys. 42, 125101 (2015)

[118] J.J. Li, J. Margueron, W.H. Long, N. Van Giai, Phys. Lett. B 753, 97 (2016)

[119] R.F. Garcia Ruiz, M.L. Bissell, K. Blaum et al., Nat. Phys. 12, 594 (2016)

[120] E. Olsen, M. Pfuetzner, N. Birge, M. Brown, W. Nazarewicz, A. Perhac, Phys. Rev. Lett. 110, 222501 (2013); ibid., 111, 139903 (2013) [Erratum]

[121] Y. Lim, X. Xia, Y. Kim, Phys. Rev. C 93, 014314 (2016)

[122] B. Zhou, Y. Funaki, H. Horiuchi, Z. Ren, G. Roepke, P. Schuck, A. Tohsaki, C. Xu, T. Yamada, Phys. Rev. Lett. 110, 262501 (2013)

[123] W.B. He, Y.G. Ma, X.G. Cao, X.Z. Cai, G.Q. Zhang, Phys. Rev. Lett. 113, 032506 (2014)

[124] T. Suhara, Y. Funaki, B. Zhou, H. Horiuchi, A. Tohsaki, Phys. Rev. Lett. 112, 062501 (2014)

[125] P.W. Zhao, N. Itagaki, J. Meng, Phys. Rev. Lett. 115, 022501 (2015)

[126] M. Girod, P. Schuck, Phys. Rev. Lett. 111, 132503 (2013)

[127] J.P. Ebran, E. Khan, T. Nikšić, D. Vretenar, Nature 487, 341 (2012)

[128] J.P. Ebran, E. Khan, T. Nikšić, D. Vretenar, Phys. Rev. C 89, 031303(R) (2014)

[129] S.G. Zhou, Constraint cluster structure from covariant density functional theory (2015), talk given at the SINAP-CUSTIPEN Workshop on Clusters and Correlations in Nuclei, Nuclear Reactions and Neutron Stars, Dec. 14-18, 2015, Shanghai, China,

http://sinap-custipen2015.csp.escience.cn/

[130] J. Dobaczewski, J. Phys. G: Nucl. Part. Phys. 43, 04LT01 (2016)

[131] S.H. Shen, J.N. Hu, H.Z. Liang, J. Meng, P. Ring, S.Q. Zhang, Chin. Phys. Lett. 33, 102103 (2016)

[132] J.R. Stone, P.A.M. Guichon, P.G. Reinhard, A.W. Thomas, Phys. Rev. Lett. 116, 092501 (2016)

[133] X.H. Zhou, High intensity heavy ion accelerator facility (HIAF) and its physics goals (2016), talk given at the CUSTIPEN-IMP-PKU Workshop on Physics of Exotic Nuclei, Dec. 12-15, 2016, Huizhou, China,

http://162.105.147.5/2016_custipen/pre_downloads/ppt/12DecMor/1.pdf

[134] I. Tanihata, What physics can we study with HIAF's high-energy beams (2016), talk given at the CUSTIPEN-IMP-PKU Workshop on Physics of Exotic Nuclei, Dec. 12-15, 2016, Huizhou, China, http://162.105.147.5/2016_custipen/pre_downloads/ppt/new/n_1.pdf

[135] S. Zeng, W. Liu, Y. Ye, Z. Guo, Chin. Sci. Bull. 60, 1329 (2015) (in Chinese) 